Review

\title{
ENaC/DEG in Tumor Development and Progression
}

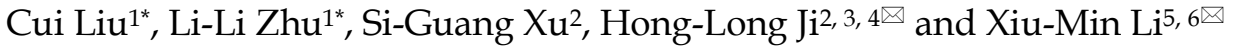 \\ 1. School of Nursing, Xinxiang Medical University, Xinxiang 453003, Henan Province, P. R. China; \\ 2. Institute of Lung and Molecular Therapy, Xinxiang Medical University, Xinxiang 453003, Henan Province, P. R. China; \\ 3. Department of Cellular and Molecular Biology, University of Texas Health Science Center at Tyler, Tyler, Texas, USA; \\ 4. Texas Lung Injury Institute, University of Texas Health Science Center at Tyler, Tyler, Texas, USA; \\ 5. Center for Cancer Research, Xinxiang Medical University, Xinxiang 453003, Henan Province, P. R. China; \\ 6. Department Gastroenterology, the Third Affiliated Hospital of Xinxiang Medical University, Henan Province, P. R. China. \\ * These authors contributed equally to this work. \\ $\bowtie$ Corresponding authors: Xiu-Min Li (email: lxm3029981@126.com) or Hong-Long Ji (email: honglong.ji@uthct.edu).
}

() Ivyspring International Publisher. Reproduction is permitted for personal, noncommercial use, provided that the article is in whole, unmodified, and properly cited. See http://ivyspring.com/terms for terms and conditions.

Received: 2016.03.30; Accepted: 2016.06.29; Published: 2016.09.13

\begin{abstract}
The epithelial $\mathrm{Na}^{+}$channel/degenerin $(\mathrm{ENaC} / \mathrm{DEG})$ superfamily, including the acid-sensing ion channels (ASICs), is characterized by a high degree of similarity in structure but highly diverse in physiological functions. These ion channels have been shown to be important in several physiological functions of normal epithelial cells, including salt homeostasis, fluid transportation and cell mobility. There is increasing evidence suggesting that ENaC/DEG channels are critically engaged in cancer cell biology, such as proliferation, migration, invasion and apoptosis, playing a role in tumor development and progression. In this review, we will discuss recent studies showing the role of $\mathrm{ENaC}$ and $\mathrm{ASIC}$ channels in epithelial cells and its relationship to the oncogenesis.
\end{abstract}

Key words: ENaC, ASIC, proliferation, migration, apoptosis, cancer cells.

\section{Introduction}

Epithelium is the most common component of human tissues, which mainly functions in body protection, regulation of fluid homeostasis and exchange, and sensation of external stimuli $(1,2)$. The homeostatic balance between cell regeneration and apoptosis of epithelial cells is under precise control or regulation. Therefore, unrestricted proliferation or apoptosis of epithelial cells can lead to abnormal status and even diseases. Recent studies have revealed that various epithelial ion channels play an important role in the regulation of fluid/ion homeostasis (3). Therefore, the abnormal function in these ion channels may lead to certain congenital diseases (4-10) and human cancers (11-13).

The genes encoding the epithelial $\mathrm{Na}^{+}$ channel/degenerin (ENaC/DEG) were firstly discovered in the early 1990s. The ENaC/DEG family includes structurally related acid-sensing ion channels (ASIC). It is well known that one of the major physiological functions of the tight and polarized epithelial monolayer is to actively adjust the turnover and resolution of luminal fluid and the machinery for transepithelial salt and fluid transport is distributed in a polarity-dependent manner. Members of the ENaC/DEG family are widely expressed in epithelia including kidney (14-16), lung (17) and colon $(18,19)$. Several studies have shown that the ENaC/DEG channels are responsible for $\mathrm{Na}^{+}$ uptake and fluid transportation (20) and are necessary to maintain the $\mathrm{Na}^{+}$balance, fluid balance and homeostasis for epithelial cells. It has been also reported that the members of the ENaC/DEG family sense the mechanical stimuli and respond with membrane depolarization (21).

The origin of almost $90 \%$ of all human cancers is from abnormal epithelial cell functions. Studies have accumulated evidence to support a role for the $\mathrm{ENaC} / \mathrm{DEG}$ channels in driving malignant cancer cell behaviors and brain tumors $(22,23)$. In this review, we will discuss the expression of the $\mathrm{ENaC}$ and ASIC 
channels in tumor cells and their role in tumor development and progression, and highlights them as potential new targets for cancer therapy in the future.

\section{Role of ENaC/DEG in tumor development and progression}

Four ENaC subunits, namely $\alpha, \beta, \gamma$ and $\delta$, are encoded by the Scnn1 genes (24). The Scnn1 genes have been implicated in oncogenesis (25). ENaC subunits form heterotrimeric complexes that function as constitutively active $\mathrm{Na}^{+}$channels, with different subunit composition resulting in channels with distinct kinetics. The $\mathrm{ENaC}$ channels are widely reported to mediate the transport of $\mathrm{Na}^{+}$from the lumen into the epithelial cells, through which the cell may migrate. As discussed below, there is increasing evidence to suggest a significant role for the $\mathrm{ENaC}$ channels in tumor cells, particularly proliferation, migration and apoptosis.

\section{1. $\alpha-\mathrm{ENaC}$}

The a-ENaC has been proposed as an important mediator in the processes of cancer cell proliferation. For example, Maryna et al (26) showed an appreciable and reversible increase in the $\mathrm{Na}^{+}$conductance in human liver hepatocellular carcinoma HepG2 cells under different degree of hypertonic stress, which could be inhibited by flufenamate and amiloride. These two drugs decreased HepG2 cell proliferation. Similar to treatment with flufenamate and amiloride, knockdown of a-ENaC using small interfering RNA (siRNA) strongly reduced hypertonic stress-induced $\mathrm{Na}^{+}$currents. FACS analysis showed that silencing of a-ENaC inhibited cell proliferation, due to an increase in the G2/M phase and a decrease in the G1 phase of the cell cycle and furthermore, increased cell apoptosis. Silencing of a-ENaC led to strong reduction in the cell volume as shown using scanning acoustic microscopy.

In addition to a role in cancer cell proliferation and apoptosis, a-ENaC has been reported to be involved in promoting cancer cell migration (27-29). The a-ENaC protein expression determined by immunocytochemistry was noticeably elevated at the leading edge of wound that was introduced by scratching to choriocarcinoma BeWo cell monolayer cultured with aldosterone (27). Moreover, in wound healing assays, treatment with amiloride alone had no effect on cell migration but completely prevented aldosterone-induced increase in cell migration. Aldosterone-induced increase in cell migration was also abolished in cells treated with antisense oligonucleotides directed against $\mathrm{a}-\mathrm{ENaC}$, but not in cells treated with sense oligonucleotides. Taken together, these results provide evidence to suggest that up-regulation of a-ENaC expression by aldosterone or cancer lesion plays an important role in cancer cell migration and post-injury recovery (27). Mirshahi et al showed that cortical actin structures evinced the major factor affecting the activity of $\mathrm{ENaC}$-like channels that are insensitive to amiloride (30).

\section{Y-ENaC}

A recent study reports a role for $\gamma-\mathrm{ENaC}$ in mediating hypotonic stress-induced proliferation and apoptosis of inner medulla collecting duct (IMCD) cells (31). As determined by FACS and MTT assays, exposure of IMCD cells to hypotonic stress resulted in significant decrease in cell proliferation and increase in cell apoptosis. Such hypotonic stress-induced effects on cell proliferation and apoptosis were prevented by treatment with 11,12-epoxyeicosatrienoic acids (EET) or adenovirus-mediated overexpression of cytochrome P2C23(CYP), the predominant epoxygenase isoform responsible for EET synthesis in rat kidney. Western blotting analysis demonstrated that the $\gamma$-ENaC expression in IMCD cells was significantly up-regulated by hypotonic stress, which was prevented by treatment with EET or overexpression of CYP2C23. These observations have led to the notion that regulation of the $\gamma$-ENaC expression represents a molecular mechanism responsible for the anti-apoptotic actions of EETs in IMCD cells under hypotonic stress conditions.

\section{3. $\delta$-ENaC}

The $\delta$-ENaC expression was shown in human melanoma G-361 cells using reverse transcription-polymerase chain reaction and cell-based in situ hybridization techniques (32). The $\delta$-ENaC expression has also been documented using immunocytochemistry in human melanoma cells (32). This finding led to the proposal that $\delta$-ENaC is a novel therapeutic target for treating malignant melanoma.

\section{Role of ASICs in tumor development and progression}

In addition to $\mathrm{ENaC}$ channels, ASICs are members of the ENaC/DEG family. Four different genes are identified, encoding six subunit isoforms, termed ASIC1a, ASIC1b, ASIC2a, ASIC2b, ASIC3 and ASIC4. These subunits can assemble functional hetero/homotrimeric channels that are sensitive to inhibition by amiloride (33).

\section{ASICI}

Cheng et al (34) found that overexpression of ASIC1a was associated with tumor cell migration and 
invasion. Under hypoxic and acidic conditions, an important feature of the tumor microenvironments, the ASIC1a mRNA and protein expression was obviously reduced in human hepatocellular carcinoma SMMC-7721 cells. In contrast, the ASIC1a expression was enhanced in a moderately acidic extracellular environment (e.g., $\mathrm{pH}$ 6.5). Therefore, ASIC1a may be overexpressed in hepatocellular carcinoma tissues, which are correlated with the disease development. Silencing of the ASIC1a expression inhibited cancer cell migration and invasion.

The study by Ross al (35) identified a cation conductance in D54-MG1 glioma cells, which was absent in normal human astrocytes. This conductance was sensitive to inhibition by psalmotoxin, an ASIC1-specific peptide inhibitor, as well as amiloride, suggesting that it was mediated by ASCI1a. Regulatory volume recovery from hyperosmotic stimulus-induced cell shrinkage was inhibited by replacement of extracellular $\mathrm{Na}+$ with $\mathrm{NMDG}^{+}$or treatments of cells with amiloride and psalmotoxin. These results support a role for the ASCI1a channel in restoring cell volume during migration of human glioma cells in the brain.

\section{ASIC2}

An early electrophysiological study documented amiloride-sensitive constitutive currents in cells from high-grade human malignant glioma, but not in cells from low grade tumors or human normal astrocytes (36). In a subsequent study, the same group revealed that surface expression of ASIC2 protein was detected in normal astrocytes but completely absent in high-grade human malignant glioma, immortalized human glioma cells (37). In further experiments, it was shown that treatments with glycerol, a chemical chaperone, or sodium 4-phenylbutyrate, a transcriptional regulator, stimulated trafficking of the intracellularly localized ASIC2 protein to the plasma membrane, leading to significant increase in ASIC2 protein at the cell surface and suppression of amiloride-sensitive currents. Both treatments reduced glioma cell proliferation and migration. These results led to the hypothesis that promotion of the ASIC2 cell surface expression and down-regulation of the amiloride-sensitive channel activity may reverse high-grade glioma cells to cells with normal astrocyte phenotypes.

Vascular smooth muscle cell (VSMC) migration is important for vascular genesis and vascular remodeling after injury. A separate study showed that ASIC2 also participated in inhibiting VSMC cell migration (38). Similar to what described above in high-grade glioma cells, ASIC2 was largely retained intracellularly. Treatment with glycerol increased ASIC2 cell surface expression and inhibited platelet-derived growth factor-induced cell migration. Moreover, this study showed that siRNA-mediated silencing of heat shock protein 70 (Hsc70) promoted ASIC2 protein cell surface expression and inhibition of cell migration, which was prevented by silencing of ASIC2 expression. These results suggest that Hsc70-dependent regulation of ASIC2 surface expression is an important regulatory mechanism in the processes of vascular genesis and vascular remodeling.

\section{Conclusions and Future Perspectives}

It has been 20 years since $\mathrm{ENaC}$ was first molecularly cloned from mouse colonic epithelium. As the earlier studies on the ENaC/DEG channels predominantly focused on their physiological functions in liver, kidney and colon epithelial cells, and showed that ENaC channel function was necessary for homeostasis of water and ion. A number of recent studies have attracted attention to the expression and function of these channels in cancer cells. As discussed above, evidence is emerging to support that the ENaC/DEG channels play a role in tumor development and progression, and stimulation or inhibition of particular ion channel expression and activity may be beneficial in certain disease conditions. Evidently, more efforts are required to better inform the relationships between the $\mathrm{ENaC} / \mathrm{DEG}$ channels and tumor cells and thereby how to treat tumors by targeting these channels.

\section{Acknowledgements}

We are grateful to the predoctoral funding support from Xinxiang Medical University and the Chinese Government Scholarship for study. We thank Dr. Lin-Hua Jiang from University of Leeds for his help during revision of the manuscript and Dr. Jian Zhu for commenting on the manuscript.

\section{Grant support}

This study was supported by the National High Technology Research and Development Program of China (2012AA02A201-1), the Science and Technology Development Plan (112102310283 and 122102310198) and American Heart Association (AHA GRNT20130034).

\section{Author Contributions}

Cui Liu, Li-Li Zhu and Si-guang Xu contributed to manuscript writing, and Xiu-min $\mathrm{Li}$ and Hong-Long Ji contributed to manuscript revision. 


\section{Competing of Interest}

The authors declare that they have no competing interests.

\section{References}

1. Olaf S. The retinal pigment epithelium in visual function. Physiol Rev. 2005; 85:845-81.

2. Dubyak G R. Ion homeostasis, channels, and transporters: an update on cellular mechanisms. Adv Physiol Educ. 2004; 28:143-54.

3. Bawa B, Abbott L C. Analysis of calcium ion homeostasis and mitochondrial function in cerebellar granule cells of adult CaV 2.1 calcium ion channel mutant mice. Neurotox Res. 2008; 13:1-18.

4. Lu C, Pribanic S, Debonneville A, Jiang C, Rotin D. The PY Motif of ENaC, Mutated in Liddle Syndrome, Regulates Channel Internalization, Sorting and Mobilization from Subapical Pool. Traffic. 2007; 8:1246-64.

5. Knight K K, Olson D R, Ruifeng Z, et al. Liddle's syndrome mutations increase $\mathrm{Na}^{+}$transport through dual effects on epithelial $\mathrm{Na}^{+}$channel surface expression and proteolytic cleavage. Proceedings of the National Academy of Sciences. 2006; 103:2805-8.

6. Zhou Z, Duerr J, Johannesson B, et al. The ENaC-overexpressing mouse as a model of cystic fibrosis lung disease. Journal of Cystic Fibrosis. 2011; 10:S172-82.

7. Wagner C A, Ott M, Klingel K, et al. Effects of the Serine/Threonine Kinase SGK1 on the Epithelial $\mathrm{Na}^{+}$Channel (ENaC) and CFTR: Implications for Cystic Fibrosis. Cell Physiol Biochem. 2001; 209-18.

8. Poulsen S B, Praetorius J, Damkier H H, et al. Reducing a-ENaC expression in the kidney connecting tubule induces pseudohypoaldosteronism type 1 symptoms during $\mathrm{K}^{+}$loading. Am J Physiol Renal Physiol. 2015; 310:300-10.

9. Cary B, Anikó N F T. C. Boyd, Naray-Fejes-Toth A. Steroid-mediated regulation of the epithelial sodium channel subunits in mammary epithelial cells. Endocrinology. 2007; 148:3958-67.

10. Hong-Long Ji, Biljana Jovov, et al. The Cytosolic Termini of the $\beta$ - and $\gamma$-ENaC Subunits Are Involved in the Functional Interactions between Cystic Fibrosis Transmembrane Conductance Regulator and Epithelial Sodium Channel. Biological Chemistry. 2000; 275:27947-56.

11. Litan A, Langhans S A. Cancer as a channelopathy: ion channels and pumps in tumor development and progression. Front Cell Neurosci. 2015; 9:86.

12. Rao V R, Perezneut M, Kaja S, et al. Voltage-gated ion channels in cancer cell proliferation. Cancers (Basel). 2015; 7:849-75

13. Florian L, Christos S. Ion channels in cancer: future perspectives and clinical potential. Philos Trans R Soc Lond B Biol Sci. 2014; 369:20130108.

14. Masilamani S, Kim G H, Mitchell C, et al. Aldosterone-mediated regulation of $\mathrm{ENaC}$ alpha, beta, and gamma subunit proteins in rat kidney. J Clin Invest. 1999; 104:R19-23.

15. Rajeev R, Andrew G, Burrow C R, et al. Na transport in autosomal recessive polycystic kidney disease (ARPKD) cyst lining epithelial cells. J Am Soc Nephrol. 2003; 14:827-36

16. Chiz-Tzung C, Cheng-Chieh H, Yung-Chang C, et al. Transforming growth factor-beta1 decreases epithelial sodium channel functionality in renal collecting duct cells via a Smad4-dependent pathway. Nephrol Dial Transplant. 2008; 23:1126-34.

17. Gail O, Bijan R, Michael H, et al. Oxygen and glucocorticoids modulate alphaENaC mRNA translation in fetal distal lung epithelium. Am J Respir Cell Mol Biol. 2006; 34:204-12.

18. Saxena S, Singh M, Engisch $K$, et al. Rab proteins regulate epithelial sodium channel activity in colonic epithelial HT-29 cells. Biochem Biophys Res Commun. 2005; 337:1219-23.

19. Saxena S K, Madhurima S, Hiroshi S, et al. Rab4 GTP/GDP modulates amiloride-sensitive sodium channel $(\mathrm{ENaC})$ function in colonic epithelia. Biochem Biophys Res Commun. 2006; 340:726-33.

20. Eastwood A L, Goodman M B. Insight into DEG/ENaC channel gating from genetics and structure. Physiology. 2012; 27:282-90.

21. Lang F, Pearce D. Regulation of the epithelial $\mathrm{Na}^{+}$channel by the mTORC2/SGK1 pathway. Nephrol Dial Transplant. 2016; 31:200-5.

22. Kapoor N, Lee W, Clark E, et al. Interaction of ASIC1 and ENaC subunits in human glioma cells and rat astrocytes. American Journal of Physiology Cell Physiology. 2011; 300 .

23. Vila-Carriles W H, Zhen-Hong Z, Bubien J K, et al. Participation of the Chaperone Hsc70 in the Trafficking and Functional Expression of ASIC2 in Glioma Cells. Journal of Biological Chemistry. 2007;282:34381-91.

24. Kellenberger S, Schild L. Epithelial sodium channel/degenerin family of ion channels: a variety of functions for a shared structure. Physiological Reviews. 2002; 82:735-67.

25. Li X M, Wang H, Zhu L L, et al. Genes Regulating Epithelial Polarity Are Critical Suppressors of Esophageal Oncogenesis. Journal of Cancer. 2015;6:694-700.

26. Maryna B, Tongiu L, Elmar E, et al. alpha-ENaC is a functional element of the hypertonicity-induced cation channel in HepG2 cells and it mediates proliferation. Pflugers Arch. 2009; 458:675-87.

27. Mónaco S M D, Marino G I, Assef Y A, et al. Cell migration in BeWo cells and the role of epithelial sodium channels. J Membr Biol. 2009; 232:1-13.
28. Marino G I, Assef Y A, Kotsias B A. The migratory capacity of human trophoblastic BeWo cells: effects of aldosterone and the epithelial sodium channel. J Membr Biol. 2013; 246:243-55.

29. Marino G I, Kotsias B A. Cystic fibrosis transmembrane regulator (CFTR) in human trophoblast BeWo cells and its relation to cell migration. Placenta. 2014; 35:92-8.

30. Mirshahi M, Mirshahi S, Golestaneh N, et al. Demonstration of the mineralocorticoid hormone receptor and action in human leukemic cell lines. Leukemia. 2000; 14:1097-104.

31. Wang L Y, Yang Liu, Wang H M, et al. Epoxyeicosatrienoic acids attenuating hypotonic-induced apoptosis of IMCD cells via gamma-ENaC inhibition. PLoS One. 2014; 9:e94400.

32. Yamamura $\mathrm{H}, \mathrm{Ugawa} \mathrm{S}, \mathrm{Ueda} \mathrm{T}$, et al. Expression analysis of the epithelial $\mathrm{Na}^{+}$ channel delta subunit in human melanoma G-361 cells. Biochem Biophys Res Commun. 2008; 366:489-92.

33. Vallet V, Chraibi A, Gaeggeler H P, et al. An epithelial serine protease activates the amiloride-sensitive sodium channel. Nature. 1997; 389:607-10.

34. Cheng J, Qing-Hai Y, Feng-Lai Y, et al. Involvement of acid-sensing ion channel 1alpha in hepatic carcinoma cell migration and invasion. Tumour Biol. 2015; 36:4309-17.

35. Ross S, Fuller C J, Benos D, et al. Amiloride-sensitive $\mathrm{Na}^{+}$channels contribute to regulatory volume increases in human glioma cells. Am J Physiol Cell Physiol. 2007; 293:C1181-85.

36. Vila-Carriles W H, Gergely Gy K, Biljana J, et al. Surface expression of ASIC2 inhibits the amiloride-sensitive current and migration of glioma cells. J Biol Chem. 2006; 281:19220-32.

37. Berdiev B K, Jiazeng X, Lee Anne $M$, et al. Acid-sensing ion channels in malignant gliomas. J Biol Chem. 2003; 278:15023-34.

38. Grifoni S C, Mckey S E, Drummond H A. Hsc70 regulates cell surface ASIC2 expression and vascular smooth muscle cell migration. Am J Physiol Heart Circ Physiol. 2008; 294:H2022-30. 